\title{
Il disegno della città ideale: Cosmopolis
}

The representations of the ideal town: Cosmopolis

\section{Cristina Boido}

Politecnico di Torino, Turin, Italy, cristina.boido@polito.it

\begin{abstract}
In 1548, under the Florentine lordship of the Medici, Charles V gave Cosimo I de Medici the task of defending the territories of Elba and the commercial traffic of the Tyrrhenian Sea. The Duke, who strongly believed in the potential of the island and wanted to transform it into the center of Florentine rule over the Tyrrhenian, decided to fortify the ancient city of Ferraia, the current Portoferraio. A real jewel of military town planning that took the name of Cosmopolis was born by the architect Giovanni Battista Bellucci and by the engineer Giovanni Camerini. Thanks to its natural conformation, the gulf of Portoferraio protected on one side a strip of land that closes the port like a spiral, and on the other hand protected by two rocky headlands overlooking the sea, was extremely strategic and suitable for defense. Fort Stella and Fort Falcone were built in the upper part of the promontory and the Linguella tower, near the dock, all connected by a bastion wall. Later the defense was further strengthened by walls and ramparts also on the land front side according to the project of the architect Bernardo Buontalenti, transforming the city into an impregnable fortress, as well as a safe naval base.

The study of urban representations of the city testifies to how the foundation of Cosmopolis for the Medici duchy was an event of extraordinary value, symbol of the strength of the Duke and his expansive abilities, symbol of an ideal city not only conceived and designed in contemporary treatises, but actually made.
\end{abstract}

Keywords: Ideal town, representation, Portoferraio.

\section{Introduzione}

A metà del XVI secolo Cosimo I de' Medici "è nel fiore dell'età e dell'energia. [...] I vecchi confini non bastano già più all' ambizione sempre crescente del giovane duca. Lo sguardo di lui si volge perciò al di là delle frontiere, mirando agli stati minori della Toscana, come Piombino, Siena, Lucca, i feudi dei Malaspina di Lunigiana" (Spini, 1940, p. 73). È certamente la signoria di Piombino, che comprendeva l'Elba e le altre isole con le loro pertinenze, il luogo in cui si concentrano le mire del Duca. Ottenuto ai primi di aprile del 1548 il consenso imperiale di Carlo V di Spagna, non senza grandi difficoltà, Cosimo viene autorizzato a iniziare $\mathrm{i}$ lavori di fortificazione del porto di Ferraia, per timore che "quel sito potrebbe essere facilmente occupato et con poco numero di gente, o dal Papa o dai
Francesi o da altri, et sarebbe poi molto difficile il recuperarlo" (ASF, Mediceo del Principato, 11, c. 44). Il Duca dapprima appronta opere a carattere campale, la cui esecuzione viene affidata a Pirro Colonna, capo militare dell'impresa, a Otto Di Montaguto, comandante dei guastatori, e all'architetto Giovanni Battista Bellucci. Vengono così approntati i tracciati planimetrici delle opere fortilizie sui due promontori, il forte Falcone e il forte Stella. Successivamente interviene, in sostituzione del Bellucci, l'ingegner Giovanni Camerini che completa il progetto e la realizzazione della città.

Sotto la sua guida vengono ultimati e trasformati da architetture campali in permanenti i due forti già tracciati, vengono progettati e realizzati la 
torre della Linguella in prossimità della darsena, la cinta muraria e il tessuto interno l'abitato urbano (Fara, 1997, p. 3). Dubbia e meticolosamente ricostruita risulta essere la paternità della costruzione di Portoferraio, riferita per alcuni a Giovanni Battista Bellucci, per altri a Giovanni Camerini (Battaglini, 1978, pp. 18-37). Sicuramente la costruzione della città è il risultato della progettazione e dell'attività di cantiere di entrambi, lavoro che sarà concluso nel 1558 da Gabrio Serbelloni per il completamento del circuito fortificato e successivamente, dopo la morte del Camerini, da Bernardo Buontalenti.

\section{I progetti delle fortezze}

I primi disegni relativi ai tracciati dei forti Stella e Falcone risalgono al giugno 1548 quando l'architetto Bellucci, fra le righe di una relazione sullo stato dei lavori destinata a Cosimo de Medici, schizza la forma definitiva delle prime opere difensive. Si sta infatti procedendo da un lato nei lavori di muratura del forte Stella (Fig. 1), a tracciato tanagliato, dall'altro si iniziano gli scavi di fondazione del forte Falcone con la parte della forbice (Fig. 2).



Fig. 1. Schizzo di Giovanni Bellucci relativo al fronte tanagliato del forte Stella (Archivio di Stato di Firenze, Mediceo del Principato, 388, c. 224r.).

Si tratta di architetture ancora in parte provvisorie, che diventeranno permanenti solo con l'arrivo in cantiere dell'ingegner Camerini. Considerato per l'epoca il progettista militare più famoso di Toscana, sotto la sua direzione vengono completati l'articolazione interna del forte Stella e definita la forma del portale in blocchi di pietra, sovrastato dallo stemma mediceo. Inoltre viene completata l'intera fortezza del Falcone e progettata la torre ottagonale della Linguella in marmo e mattoni. I disegni di rilievo, redatti nel
1552 dal Bellucci, in occasione della compilazione di un atlante delle più importanti città fortificate d'Europa, indicano lo stato di avanzamento del cantiere dei due forti principali, ormai praticamente completati (Figg. 3 e 4). Nei primi anni dell'Ottocento, gli elaborati di rilievo redatti dagli ingegneri di Napoleone, testimoniano la solidità e l'immutabilità dell'impianto cinquecentesco, ancor oggi quasi integralmente conservato.

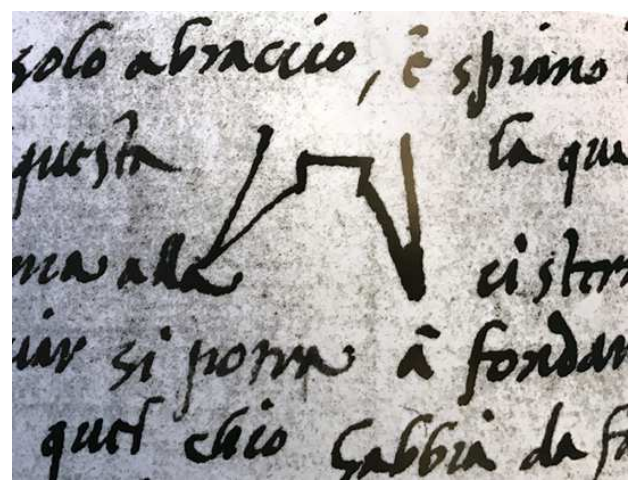

Fig. 2. Disegno del tracciato del fronte bastionato del forte Falcone, ipotizzato dal Giovanni Bellucci nel 1548 (Archivio di Stato di Firenze, Mediceo del Principato, 388 , c. 224 r.).

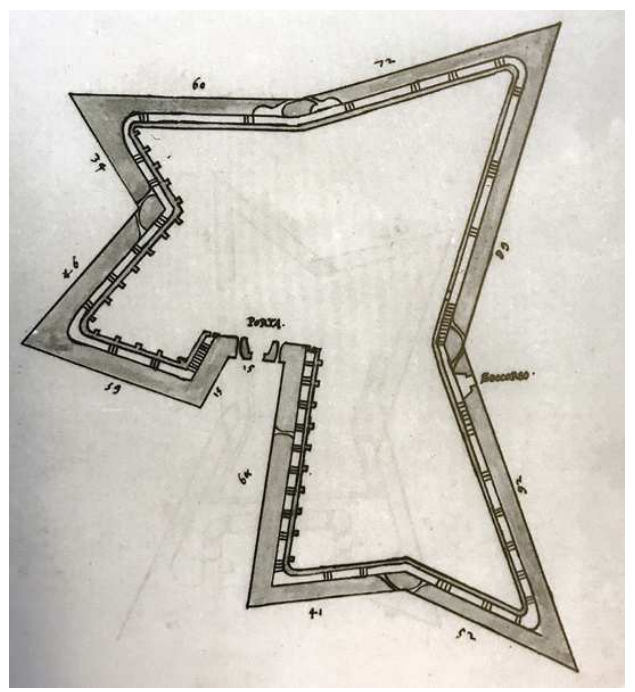

Fig. 3. Il forte Stella in un rilievo del 1552 redatto da Giovanni Bellucci (Biblioteca Nazionale Centrale di Firenze, II.I.280, c. 23r.). 


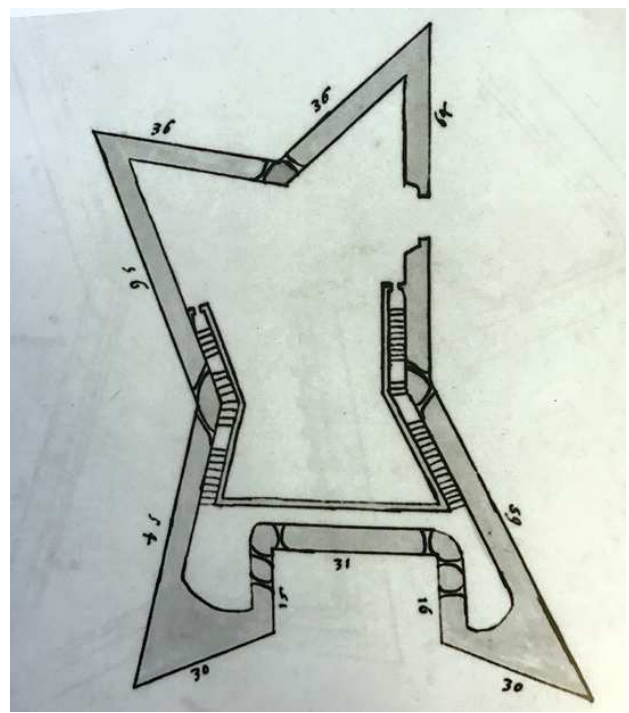

Fig. 4. Rilievo di Giovanni Bellucci riguardante la pianta del forte Falcone nel 1552 (Biblioteca Nazionale Centrale di Firenze, II.I.280, c.23r.).

\section{I disegni tecnici della città fortificata}

E' del 1549 il primo piano urbanistico generale di Cosmopolis. In realtà due sono le proposte approntate dal Camerini, come viene descritto nella lettera di accompagnamento inviata al duca Cosimo. I progetti, non identificati, hanno in comune il tracciato regolare ortogonale della maglia viaria e la centralità della piazza d'Armi, polo civile, religioso e naturalmente militare, su cui si affacciano il duomo e la residenza granducale. Un disegno successivo, redatto dall'architetto mediceo nel 1553, in occasione dei lavori di costruzione del porto, riprende $\mathrm{i}$ contenuti del piano del 1549 (Fig. 5). Qui oltre ad essere evidenziati i tratti di fondazione del muro del porto in costruzione, vengono rappresentati la cinta fortilizia, i forti, il tracciato d'impianto della rete infrastrutturale, la piazza d'Armi e alcuni edifici fra cui, probabilmente, l'antico ospedale e l'edificio delle Conserve.

Sicuramente l'intero impianto riflette la volontà di creare una città militare. La maglia viaria ortogonale consente infatti di effettuare un veloce e agile spostamento delle truppe da un fronte all'altro. Tuttavia due sono gli andamenti del tracciato per seguire in modo più perpendicolare possibile la conformazione della darsena: impianto ancor oggi facilmente riconoscibile nel tessuto urbano attuale. Dalla piazza principale, progettata originariamente quasi quadrata e ampliata nel corso della sua realizzazione, si diramano le arterie militari principali, in direzione della porta a Mare e del forte Stella, residenza del Duca e centro del potere militare. Su modello della trattatistica militare coeva, sono tracciate solo le arterie principali di attraversamento della città, rimandando in un secondo momento la suddivisione dei grandi isolati in lotti minori. Inoltre secondo i dettami della cultura militare dell'epoca, in particolare seguendo le teorie di Giuliano da Sangallo, la porta di Terra viene collocata a ridosso di un mezzo baluardo per poter essere più facilmente protetta.

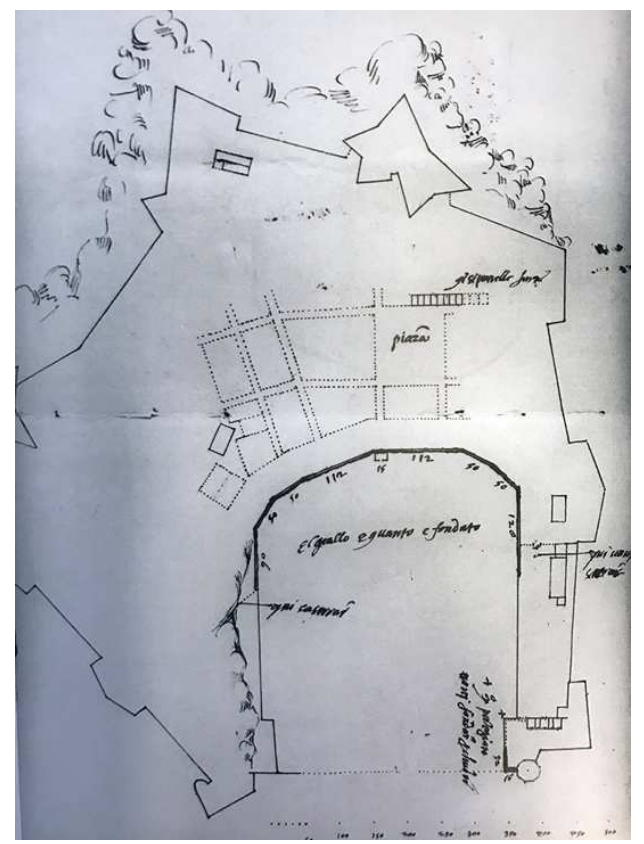

Fig. 5. Piano urbanistico di Cosmopolis elaborato da Giovanni Camerini nel 1549, riportato in un disegno di progetto del 1553 (Archivio di Stato di Firenze, Mediceo del Principato, 417A, cc. 989v-990r.).

Anche il Bellucci nello stesso periodo redige un disegno della città (Fig. 6). Si tratta di un rilievo eseguito nel 1552 quando l'architetto torna a Portoferraio per illustrare la fortezza urbana in costruzione ed inserirla in quell'atlante di città fortificate che stava predisponendo per il Duca mediceo. La mappa descrive la conformazione orografica del territorio, l'invaso del golfo, il tracciato del circuito fortilizio, in parte realizzato ed in parte ipotizzato, soprattutto il tratto settentrionale, disegnato a tratteggio, l'impianto dei 
forti ormai conclusi, mentre nulla viene delineato sulla parte interna all'abitato.

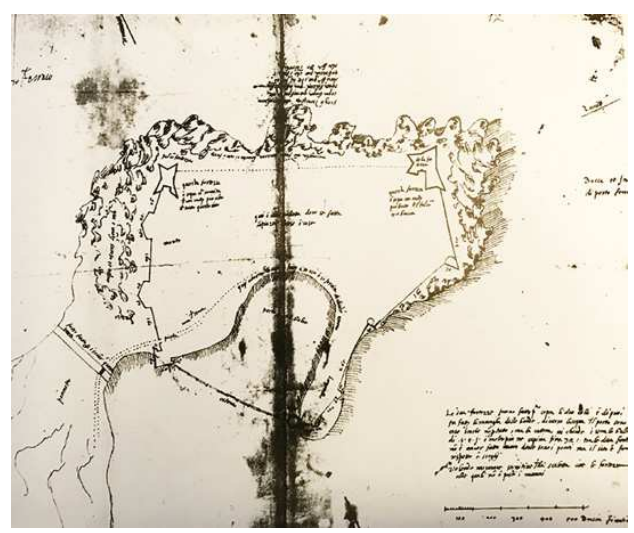

Fig. 6. Rilievo planimetrico della cittàfortezza di Portoferraio redatto da Giovanni Bellucci nel 1552 (Biblioteca Apostolica Vaticana, Barberiniano Latino).

La planimetria tuttavia con viene inclusa nella pubblicazione, forse perché i lavori non sono ancora completati e la città non poteva ancora essere considerata fra le più importanti centri fortificati d'Europa.

Negli anni successivi, compiuto e reso ancor più sicuro il circuito fortificato, grazie anche all'intervento dell'ingegner Gabrio Serbelloni, già consulente di Cosimo per le fortificazioni di Pisa e Cortona, ci si concentra nella realizzazione di quegli edifici pubblici necessari al funzionamento logistico della città. L'ingegner Camerini è chiamato a progettare, oltre che il mulino, l'ospedale, la Biscotteria, cioè la fabbrica dei forni laddove si cuoceva il biscotto dei militari, il Duomo, il convento e la chiesa di San Francesco, il Santissimo Sacramento, l'arsenale. Si tratta di architetture per cosi dire spartane, ma tecnicamente raffinate, soprattutto per quanto riguarda gli impianti idrici, così determinanti per il funzionamento di una città fortezza, costruzioni ideate secondo il rigore geometrico tipico di un ingegnere militare, dove la funzionalità risultava essere il principale criterio della progettazione.

Nel 1575 la planimetria della cinta muraria rappresentata da Matteo Neroni (Fig. 7) riporta le modifiche del nuovo fronte di terra progettato da Bernardo Buontalenti. Arrivato a Portoferraio dopo la morte del Camerini, avvenuta nel 1570, l'architetto, chiamato a migliorare il funzionamento logistico della città, completando l'arsenale e costruendo numerosi mulini, si occupa di potenziare il fronte occidentale della piazzaforte. Inglobando l'antico impianto del Camerini, vengono realizzate nuove cortine parallele alle facce dei baluardi creando un nuovo e inespugnabile sistema difensivo, ispirato ad alcuni progetti di Antonio da Sangallo il Giovane, ideale e perfettamente confacente alla ideale e perfettamente confacente alla conformazione topografica del promontorio portoferraiese (Fara, 1997, p. 21).

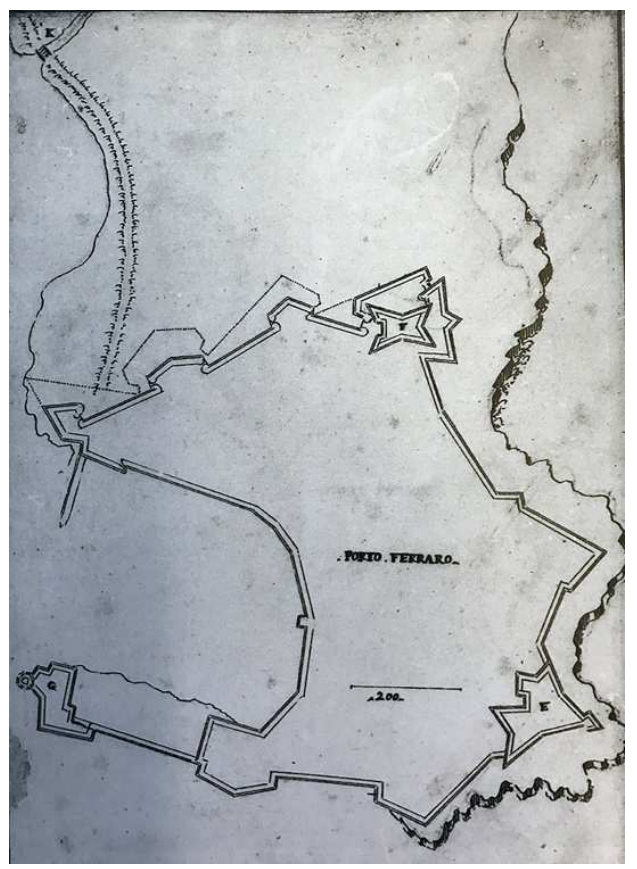

Fig. 7. Disegno del circuito fortificato rappresentato da Matteo Negroni nel 1575 in cui viene raffigurato il nuovo fronte di terra progettato da Bernardo Buonatalenti (Istituto Storico e di Cultura dell'Arma del Genio di Roma, Biblioteca, 36.B.51, c. 98).

\section{Le rappresentazioni celebrative urbane}

E' del 1553, circa, la prima rappresentazione tridimensionale della città (Fig. 8). Viene probabilmente redatta da un collaboratore del Camerini e ben testimonia l'andamento orografico del terreno su cui si sta costruendo Cosmopolis. Il punto di vista dell'osservatore risulta posizionato a sud est della città, ad un livello leggermente superiore rispetto il forte Falcone, luogo altimetricamente più elevato della città. Questo permette di illustrare al meglio l'intero sistema di difesa urbano fornendo un disegno dettagliato 


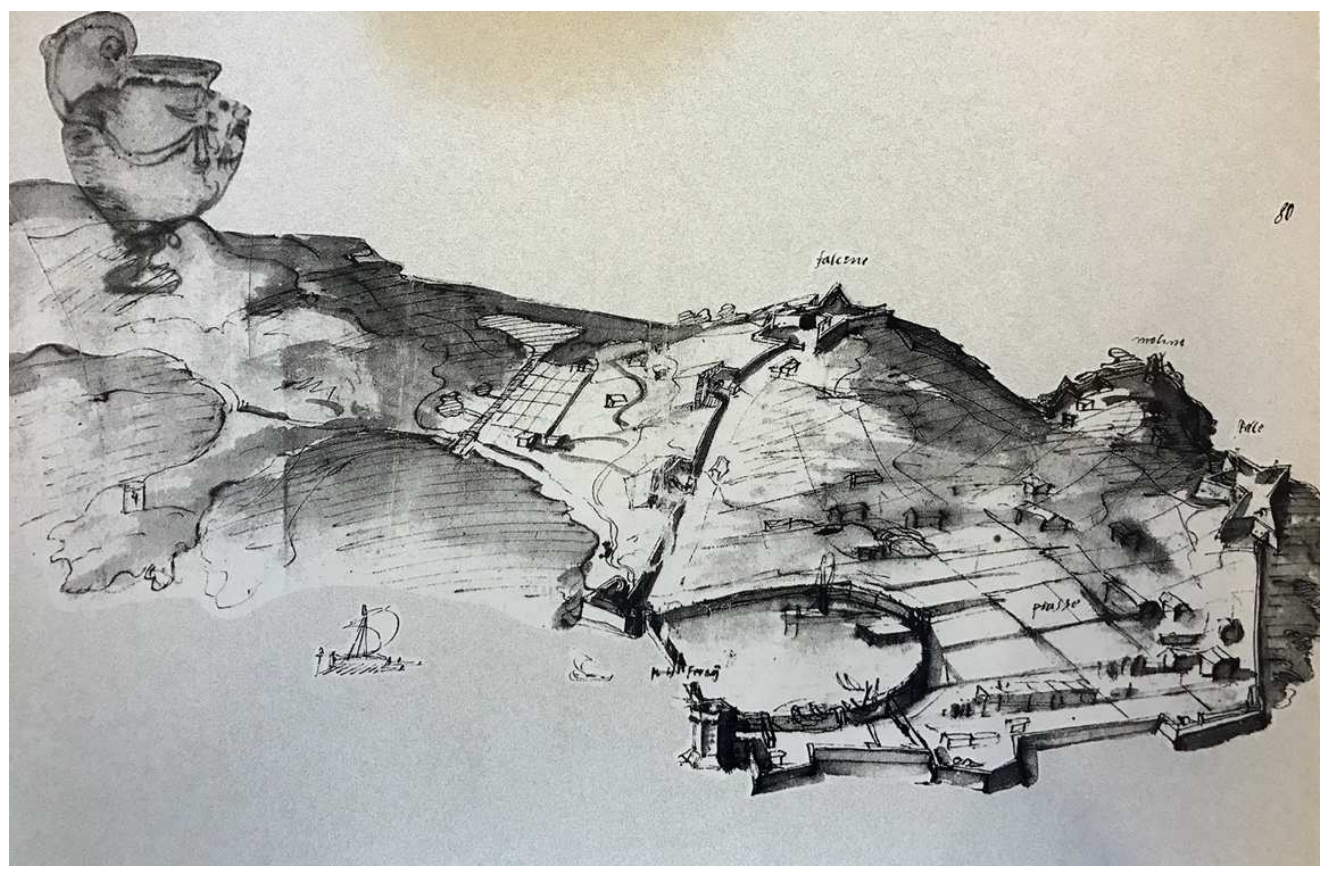

Fig. 8. Veduta acquerellata raffigurante Portoferraio realizzata probabilmente da un collaboratore di Giovanni Camerini nel 1553 (Biblioteca Comunale di Siena, S.II.7, c. 80r/v.).

del fronte di terra e di quello a mare ad est della città. La veduta evidenzia la cinta delle mura caratterizzata dai forti principali, dai baluardi intermedi, dai contrafforti, dai terrapieni, dalle porte e dalla catena del porto, a protezione della rada. Spiccano nella rappresentazione i forti Falcone e Stella, la torre della Linguella e il mezzo baluardo dei Mulini, che inglobava l'edificio dove era stata collocata una macina donata dal Duca. Viene indicato l'impianto ortogonale degli isolati previsti dal progetto e vengono disegnati più in dettaglio i pochi edifici preesistenti.

Sicuramente di grande valore celebrativo risultano essere le raffigurazioni della città presenti sulle due medaglie fatte coniare nel 1555 e nel 1569 da Cosimo appositamente per celebrare la fondazione di Portoferraio (Battaglini, 1978, p. 43). Nonostante l'essenzialità e la schematicità della rappresentazione si possono riconoscere le strutture difensive connotanti la cittàfortezza: prima di tutto la catena della darsena, elemento enfatizzato e predominante nella rappresentazione, e a seguire sulla sinistra la Porta di Terra, e, a salire, i due baluardi connotanti la cortina muraria che conduce al forte Falcone, ormai compiuto, e poi ancora il forte Stella, da cui riparte la cinta che giunge fino al mare con la torre della
Linguella. Da sottolineare la mancanza di qualsiasi opera difensiva nella parte settentrionale della città, tra i due forti principali, quasi a sottolineare come la scoscesa difesa naturale.

Questo anche testimoniato dal disegno di rilievo del 1552 del Bellucci, che, scarpata verso il mare risultasse di per sé una come si è già accennato, tracciava con una linea tratteggiata il profilo settentrionale della cortina muraria, completata solo successivamente. Ma senza dubbio la raffigurazione celebrativa per eccellenza che commemora la fondazione della nuova città ducale all'Elba è la veduta dipinta da Giorgio Vasari nell'affresco della sala di Cosimo I in Palazzo Vecchio a Firenze (Figg. 9 e 10). Vengono rappresentati sulla sinistra in primo piano Cosimo, con alle spalle l'ingegner Camerini, Luca Martini, il provveditore di Pisa o più probabilmente provveditore delle fortezze di Portoferraio, e il segretario Lorenzo Pagni, oltre che il nano di corte Morgante. Il Duca tiene nella mano sinistra la mappa di Cosmopoli, mentre con la destra indica la città raffigurata in secondo piano sulla destra del dipinto.

Qui il punto di vista dell'osservatore è posto molto in alto e angolato verso levante: ne deriva 
un'immagine altimetricamente falsata, ma topograficamente corretta rispetto alla pianta di $\mathrm{Ca}-$ merini, di cui sicuramente il Vasari si serve nell'impostazione del dipinto. Come aveva già sperimentato per la rappresentazione di Firenze raffigurata durante l'assedio degli anni Trenta del Cinquecento, Vasari utilizza una carta planimetrica urbana in combinazione di una bussola per costruirne l'immagine tridimensionale. Tutto ciò rende la veduta non solo celebrativa, ma, a parte qualche imprecisione, precisa e rispondente nelle proporzioni delle varie parti della città.

Raffigurata in una situazione probabilmente tra la fine degli anni Cinquanta e gli inizi degli anni Sessanta, "l'isola dell'Elba con Portoferraio, e le fortezze della Stella, e del Falcone edificate da S.E., che l'ho ritratte là nel lontano con tutte quelle strade e mura che per l'appunto vi sono" (Vasari, 1906, p. 191), la città risulta essere planimetricamente definita. Emerge la cortina muraria sia esterna che interna alla darsena completamente ultimata, con la catena a protezione della baia e la porta a Mare e spiccano, acquerellati in rosa, le due fortezze e la torre della Linguella. Sono definiti da una rete viaria pressoché ortogonale i grandi isolati secondo cui viene orga- nizzato il tessuto urbano, mentre vengono disegnati con elementi architettonici di dettaglio gli edifici già realizzati. Nonostante qualche approssimazione nel disegno della maglia planimetrica e qualche inesattezza nel tracciato del forte Falcone, rappresentato senza fronte bastionato, la veduta risulta senza dubbio l'apoteosi della magnificenza del Duca mediceo, dominatore dell'isola d'Elba.

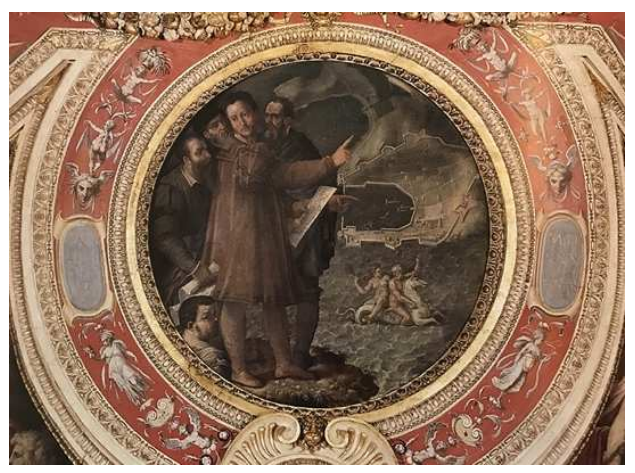

Fig. 9. Affresco di Giorgio Vasari a Palazzo Vecchio a Firenze raffigurante Cosimo I e la nuova città di Cosmopolis.

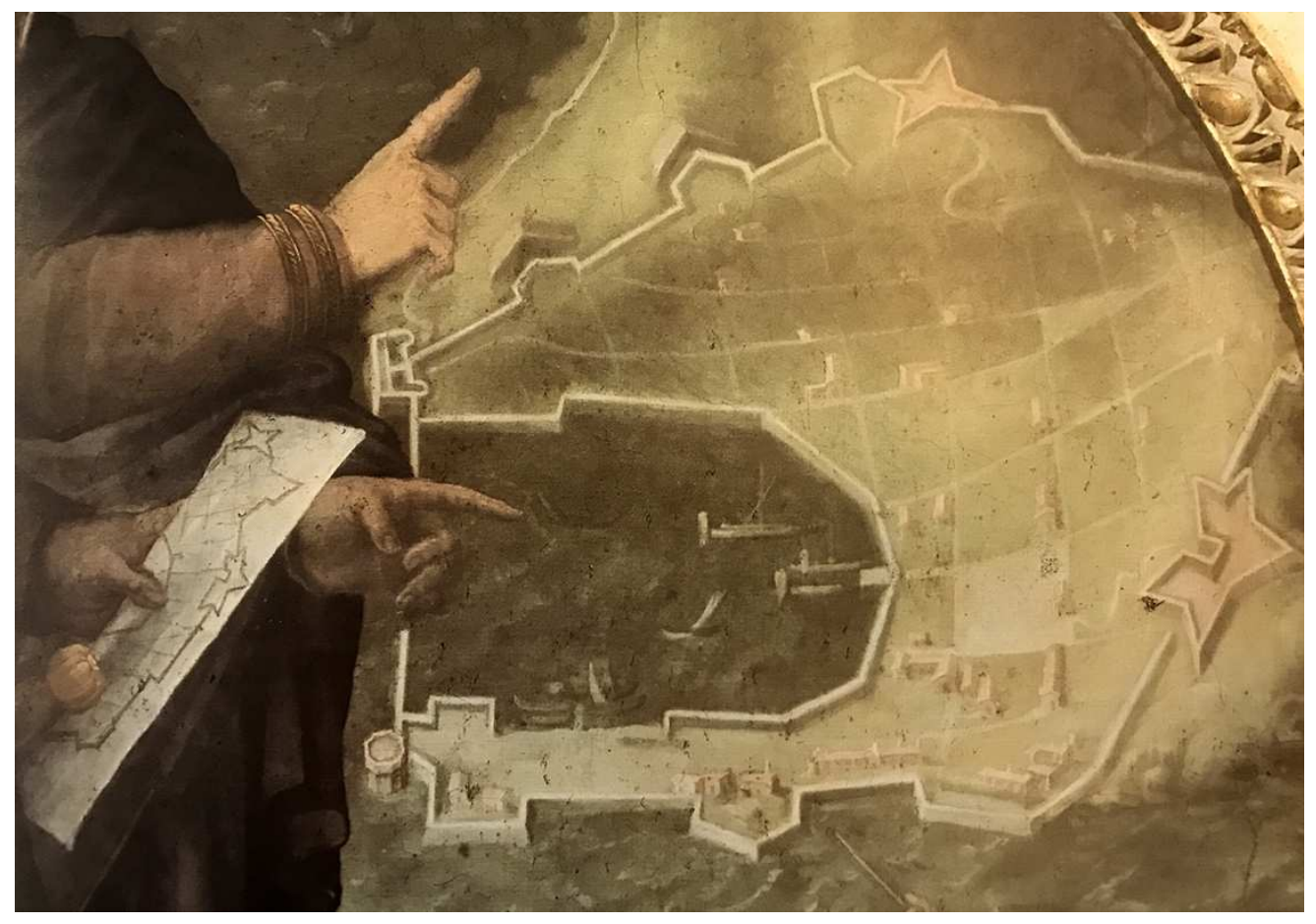

Fig. 10. Particolare del dipinto del Vasari che descrive in dettaglio l'impianto urbano di Portoferraio. 


\section{Conclusioni}

L'analisi delle rappresentazioni urbane di Portoferraio a metà Cinquecento testimonia come per il ducato di Cosimo I la fondazione di Cosmopolis sia un avvenimento di straordinario valore, emblema della forza del Duca e delle sue abilità espansive. Si tratta di una fra le poche città ideali non solo pensata e progettata sulla carta, ma realmente realizzata, a differenza di molti altri esempi descritti nella trattatistica coeva, dove si mettono in pratica quei caratteri di funzionalità, ordine razionale ed equilibrio fondamentali per la creazione di inespugnabili piazzaforti militati, care alla cultura rinascimentale.

La città di Portoferraio, sia nell'innovativo e particolare impianto insediativo e fortilizio, sia nel nome stesso che le viene attribuito, rispecchia la volontà di celebrare il potere del Duca: Cosmopolis, la città di Cosimo. In effetti risulta, il nome stesso, un elemento di propaganda del potere ducale: mai il Duca nelle lettere, o anche in atti ufficiali come gli Statuti, utilizza tale appellativo, descrivendo l'abitato sempre con il nome di Portoferraio.

Attraverso le testimonianze cartografiche qui presentate, da un lato i disegni bidimensionali di progetto o di rilievo, dell'altro le immagini tridimensionali più iconografiche, è possibile delineare quando anche la rappresentazione concorresse, e talvolta fosse imprescindibile, nel diventare strumento divulgativo e celebrativo nel magnificare la grandezza di una città o di un Principe.

\section{Bibliography}

Battaglini, G.M. (1978). Cosmopolis Portoferraio medicea. Storia urbana 1548-1737, Multigrafica Ed., Roma.

Battaglini, G.M. (1980). "Portoferraio", in Livorno: progetti e storia di una città tra il 1500 e il 1600, Nistri-Lischi e Pacini Ed., Pisa, pp. 309-340.

Fara, A. (1997). Portoferraio. Architettura e Urbanistica 1548-1877, Fondazione Giovanni Agnelli Ed., Torino.

Foresi, E. (1899). L'Isola d'Elba, Paggi Ed., Pitigliano.

Manetti, R. (1995). Portoferraio e le sue antiche fortificazioni, Il Libraio Ed., Portoferraio.

Spini, G. (1940). Cosimo I de'Medici, Lettere, Vallecchi Ed., Firenze.

Vasari, G. (1906). Le vite de' più eccellenti pittori scultori ed architettori, Sansoni Ed., Firenze, tom. VIII, p. 191. 
